\title{
Single Track Ultra-short Pulsed Laser Ablation on Additive Manufactured Metallic Surfaces
}

\author{
Simon Ruck ${ }^{* 1,2}$, David K Harrison ${ }^{2}$, Anjali De Silva ${ }^{2}$, Max-Jonathan Kleefoot ${ }^{1}$ and Harald Riegel ${ }^{1}$ \\ ${ }^{1}$ LaserApplikationsZentrum, Aalen University, Germany \\ ${ }^{2}$ School of Computing, Engineering \& Built Environment, Glasgow Caledonian University, \\ Scotland UK \\ *Corresponding author's e-mail: simon.ruck@hs-aalen.de
}

\begin{abstract}
Additive Manufacturing (AM), also called 3D printing, have been proved as a method to offer new possibilities for the production of highly complex parts. One new interesting but yet small field of application is the 3D printing of complex optical elements. However, the functional optical performance like the grade of transmission or reflection of such optics is highly dependent on the surface quality. Thus, ultra-short pulsed laser ablation as a very precise ablation technology was investigated as a post-processing technology for AM. However, for additive manufactured parts the rough and inhomogeneous surface as well as pores make it challenging to create a homogenous ablation over a larger area. In this work, a parameter study using various laser parameters for single track ablation on additive manufactured $\mathrm{AlSi} 10 \mathrm{Mg} 0.4$ surfaces is presented. The dependence between the ablated volume, in depth and width, and the set laser parameters are discussed.
\end{abstract}

DOI: 10.2961/jlmn.2021.01.2007

Keywords: additive manufacturing, AlSi10Mg0.4, metal optics, post-processing, laser ablation, ultra-short pulsed laser, optical structures

\section{Introduction}

Additive Manufacturing (AM), also called 3D printing, have been proved as a method to offer new possibilities for the production of highly complex parts. Since the initial development of AM to produce product prototypes, the importance of AM technologies has increased contribution and importance during the last decade. Already today real products and end-use parts have been produced for a lot of various industrial applications [1]. Worldwide different companies such as "Stryker, Audi, BMW, Boeing, Ford, General Electric, Hewlett-Packard, and Nike - to name a few - as well as many small and medium-sized organizations" [2] integrated AM technologies into their production systems. By adding material to a volume, mostly done by printing layer by layer, nearly all geometries are able to be produced and therefore a big economic impact is expected [3].

One new interesting but yet small field of application is the 3D printing of complex optical elements [4]. Here, the most used materials are polymers to build transmissive optics. Common Additive Manufacturing technologies for optics are two photon polymerisation $[5,6]$, stereolithographic or inkjet based processes [4]. But the layerwise build process of large transmissive optics by using multi jet modeling or fused deposition modeling causes some disadvantages. For example the current state of art describes some limitations according the damage threshold and allowed power densities in additive manufactured optic systems due to the absorption and scattering of the transmitting light at the interface between two layers [7]. Therefore, 3D printing of reflective optics could be another approach to utilize the advantages of Additive Manufacturing, such as freedom in geometry design $[7,8]$, lightweight construction [9] and reduced system complexity [8].

Interesting materials for additive manufactured reflective optics are aluminum alloys [7,9]. Aluminum and aluminum alloys have proven a high reflectivity for a broad band of wavelengths $[10,11]$. Further, they have a very good heat conductivity, which allows a good cooling by inner cooling channels, are cheap and well investigated according their laser based additive manufacturing process. However, for additive manufactured optics the surface roughness and waviness due to the AM process are a big issue [7]. Therefore, like in nearly any AM process chain, postprocesses after the printing process are needed to finish and adjust the surface as the customer or application require [1,7] To achieve surfaces with an optical quality, mostly mechanical machining processes such as diamond turning $[12,13]$ or pad polishing [14] are used. The studying of laser material processing as a post-processing method for additive manufactured optical components is of great importance. In contrast to common mechanical post-processing methods laser machining is a contactless procedure, which is automatable and allows the surface finishing of complex surfaces. Hereby, the spatial resolution is much higher compared to diamond turning or pad polishing, due to the good focusability of the laser light. Finally, laser material processing is an environmental friendly technology, which works without any chemistry and water pollution.

One laser based post-processing method e.g. is laser polishing by melting the surface layer with high power continuous or high power pulsed lasers $[7,15,16]$.

However, since next to AM also ultra-short pulsed laser technologies gained more and more importance during the last recent years their ability as a further post-processing 
technology should be investigated. First studies to structure or flatten the surface using ultra-short laser pulses were carried out in [17-19]. As part of available post-processing methods, this work focuses on surface micro structuring with ultra-short pulsed laser ablation in order to generate wide optical structures, e.g. optical gratings, on the additive manufactured parts in further experiments.

\section{Experimental procedure}

\subsection{Test sample preparation}

The single track ablation test were performed on additive manufactured $10 \mathrm{~mm}$ x $10 \mathrm{~mm}$ cubes made of AlSi10Mg0.4. The used samples were built in a laser powder bed fusion process on a TRUMPF TruPrint 1000 multilaser machine with two fiber lasers, each $200 \mathrm{~W}$ optical output power. To avoid oxidation of the material during the manufacturing process, the process was carried out under inert gas argon. The remaining oxygen content was below $0.3 \%$. The thickness of the single layers was adjusted to $20 \mu \mathrm{m}$. Further process parameters are shown in Table 1. As building material for the cubes the powder material AlSi10Mg0.4 from ECKA Granules, particle size $20-63 \mu \mathrm{m}$ was used.

Table 1 Parameters for the AM process of AlSi10Mg0.4

\begin{tabular}{lll}
\hline Parameter & Unit & Value \\
\hline laser power & $\mathrm{W}$ & 170 \\
focal diameter & $\mu \mathrm{m}$ & 55 \\
scan velocity & $\mathrm{mm} / \mathrm{s}$ & 1700 \\
\hline
\end{tabular}

37 cubes were placed on a building platform with a diameter of $100 \mathrm{~mm}$. The distance between two single cubes was $2 \mathrm{~mm}$ to avoid heat conduction influences by surrounding cubes, see Figure 1.

The size of the cubes was chosen so that the planned parameter fields, a $4 \times 9$ matrix, where each field consist of four lines with $1 \mathrm{~mm}$ length and a line to line distance of 0.1 $\mathrm{mm}$, could be placed. Also, with this setup all ablated grooves of one parameter can be afterwards measured within one step to avoid an influence of the sample position in the measurement device.

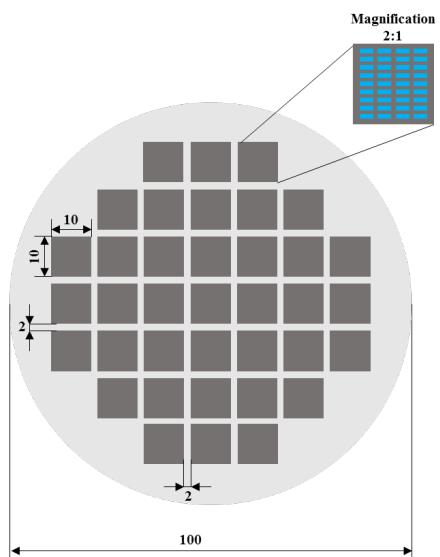

Fig. 1 Sample Layout. Additive manufactured AlSi10Mg0.4 sample cubes on the build platform. The magnification shows the placing of the parameter fields.
After the building process, the surface roughness on the top layer of the sample parts was measured with a tactile measurement device Mahr MarSurf M400 and a white light interferometer (WLI) Zygo NewView 8000. The roughness of the surface as produced (SAP) has a $R_{a}=9.79 \mu \mathrm{m}$. Since this is clearly too rough to determine ablation effects and to achieve functional optical structures with just a few microns in depth, the experiments were carried out on additionally post processed samples. Therefore, the additive manufactured samples were mechanically grinded and polished using a Struers RotoPol-31. The polishing treatment provides a very smooth surface, which is necessary to determine precisely the ablation threshold of the material, ablation depth and line width more precise. In this case a $R_{a}=0.04 \mu \mathrm{m}$ was reached.

\subsection{Structuring by direct laser ablation}

The structuring test were performed with a diode pumped laser beam source, varydisc 120 femto from Dausinger + Giesen $\mathrm{GmbH}$, Stuttgart. In this laser, a Yb:YAG disk is used as laser active medium to generate laser radiation with a center wavelength of $1030 \mathrm{~nm}$. This laser system is characterized especially by its high single pulse power of up to $600 \mu \mathrm{J}$ at $200 \mathrm{kHz}$ pulse repetition rate, the flexibility of the pulse duration from 600 fs up to $2 \mathrm{ps}$ and the option to generate the second and third harmonic of the center wavelength. The laser beam is guided across the sample parts by a galvoscanner system excelliSCAN 14 from SCANLAB GmbH. With the help of this system, it is also possible to implement high feed rates of up to $v_{\text {scan }}=15 \mathrm{~m} / \mathrm{s}$. The components were integrated in a high precision multi axes machine microcut UKP from LLT Application $\mathrm{GmbH}$, Ilmenau. Figure 2 shows the final experimental setup with the additive manufactured samples clamped onto the machine table. The exact z-position of the sample surface was determined by a camera measurement system. For the experiments, the laser focus was placed directly on the polished surface of the samples. The focal diameter $w_{f}$ of $46 \mu \mathrm{m}$ was measured using a metrolux FM100 with additional $\mathrm{M}^{2}$ module. This measurement system uses a CCD chip for detection and the second moment method to calculate the focal diameter etc. Each parameter setup was validated by using a power measurement device to check the average laser power and a photodiode to measure the pulse repetition rate before starting the experiment.

For the experiments, a wide range of parameters was varied using a full factorial design of experiments model. Table 2 lists the used laser parameters.

Table 2 Parameter range of the experiments

\begin{tabular}{llll}
\hline Parameter & & Unit & Value \\
\hline wavelength & $\lambda$ & $\mathrm{nm}$ & 1030 \\
focal diameter & $\mathrm{W}_{\mathrm{f}}$ & $\mu \mathrm{m}$ & 46 \\
number of crossings & & & 10 \\
average laser power & $\mathrm{P}_{\mathrm{av}}$ & $\mathrm{W}$ & $1-12.5$ \\
repetition rate & $\mathrm{f}_{\mathrm{rep}}$ & $\mathrm{kHz}$ & $100 ; 200$ \\
scan velocity & $\mathrm{v}_{\mathrm{scan}}$ & $\mathrm{mm} / \mathrm{s}$ & $500-2500$ \\
pulse duration & $\mathrm{t}_{\mathrm{H}}$ & $\mathrm{ps}$ & $0.8 ; 1.4 ; 2.0$ \\
\hline
\end{tabular}




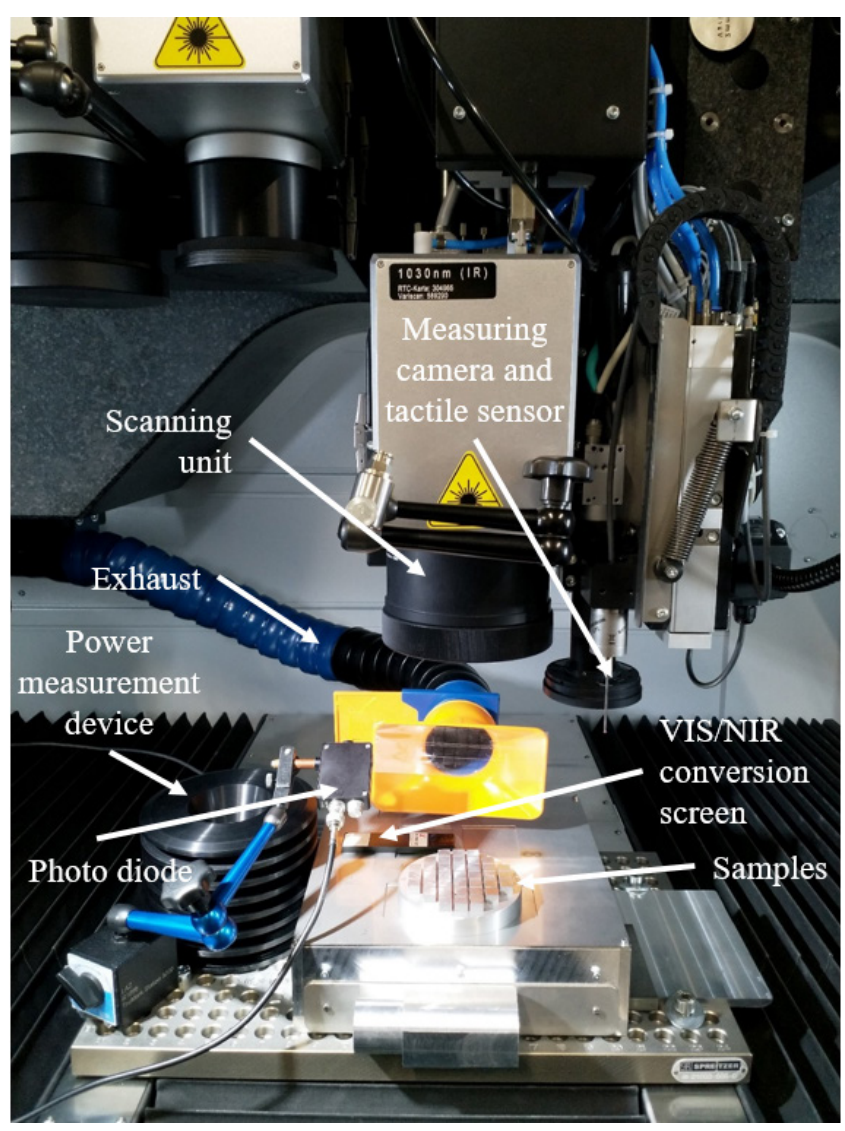

Fig. 2 Experimental setup for laser structuring

After the laser structuring, the achieved line width and depth were investigated using the Zygo NewView 8000. For this study, the ablation depth is defined as the distance between an untreated section as reference and the deepest point of the cross section of the ablated groove. The ablation width is the width of the ablated groove on the reference surface level. In addition to the measurement of the death and with also scanning electron microscope (SEM) images for selected parameters were taken using a ZEISS Crossbeam 540 to analyze the micro structured surface more closely.

\section{Experimental results}

\subsection{Impact of the fluence}

The influence of the applied fluence on the achieved ablation depth of a single line is shown in Figure 3, exemplary for the scan velocity $v_{s}$ of $1 \mathrm{~m} / \mathrm{s}$ and the pulse repetitions rate $f_{\text {rep }}=100 \mathrm{kHz}$. Equation 1, using the single pulse energy $E_{E p}$ and the enlightened area $w_{f}$, calculated the fluence $F$.

$$
F=\frac{E_{E p}}{\pi \cdot w_{f}^{2}}
$$

As increasing the fluence the ablation depth also increases.

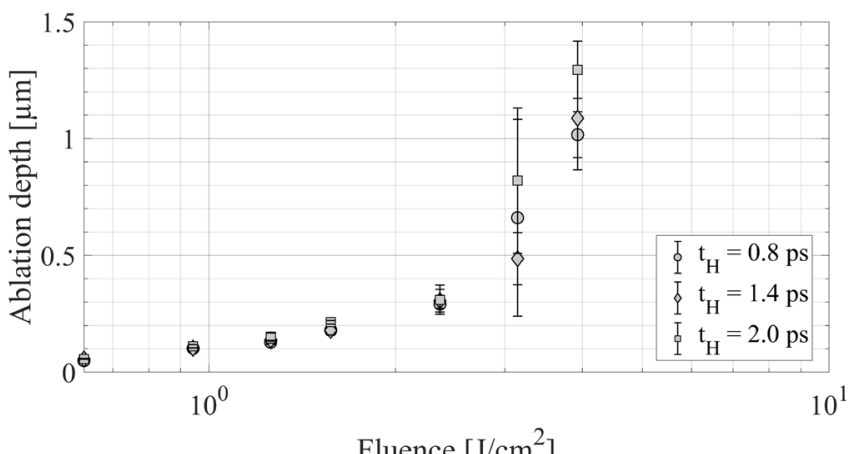

Fig. 3 Ablation depth depending on the applied fluence and pulse duration $t_{\mathrm{H}}$ for $\mathrm{a}_{\mathrm{s}}=1 \mathrm{~m} / \mathrm{s}$ and $\mathrm{f}_{\mathrm{rep}}=100 \mathrm{kHz}$

Two regimes can be observed for all scanning speeds -a regime where the optical penetration depth is dominant and a second regime where the diffusion length $l$ of the electrons into the matter is significant. In the first regime with fluences below $2.4 \mathrm{~J} / \mathrm{cm}^{2}$ the ablation depth per pulse $L$ can be described by Equation 2, where $\alpha^{-1}$ is the optical penetration depth and $F_{t h}^{a}$ the threshold fluence for this regime.

$$
L=\alpha^{-1} \cdot \ln \left(\frac{F}{F_{t h}^{a}}\right)
$$

For higher fluences in the second regime the ablation depth per pulse can be estimated using Equation 3, using the thermal diffusion length $l$ and the threshold fluence $F_{t h}^{l}$.

$$
L=l \cdot \ln \left(\frac{F}{F_{t h}^{l}}\right)
$$

In the high fluence regime, also the derivation of the measurement results increases. For deeper ablation depths pores in the material below are opened. In addition, in this regime also melt is produced, which causes further irregularities.

The correlation between a rising fluence and the achieved ablation width of the grooves is shown in Figure 4. In contrast to the ablation depth, the ablation width is not continuingly increasing with higher fluences using a constant focal position. Below $2,4 \mathrm{~J} / \mathrm{cm}^{2}$ the optical penetration depth is crucial. Here, ablation occurs just because of vaporization. A maximum is reached for the maximum focal size, if $0-100 \%$ of the intensity are taken to determine the diameter. For fluences higher $2,4 \mathrm{~J} / \mathrm{cm}^{2}$ the ablation process is also melt assisted. Therefore, the measured width lowers because of melt solidification, which reduces the width of the groove of the measured reference level.

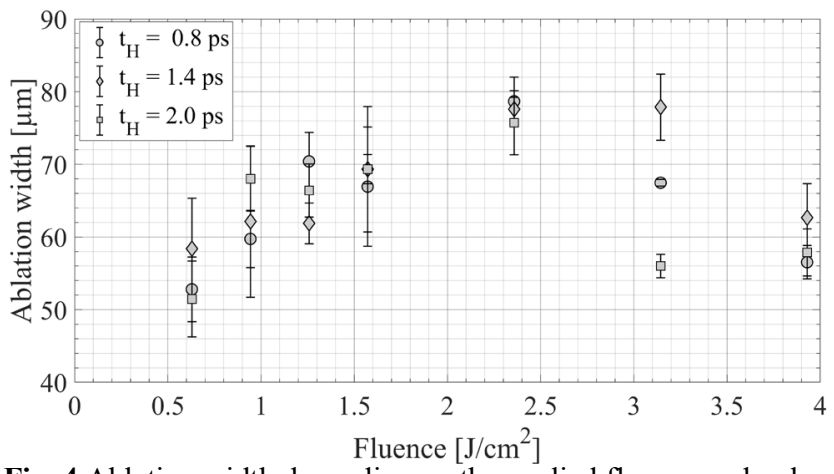

Fig. 4 Ablation width depending on the applied fluence and pulse duration $t_{\mathrm{H}}$ for $\mathrm{a}_{\mathrm{scan}}=1 \mathrm{~m} / \mathrm{s}$ and $\mathrm{f}_{\text {rep }}=100 \mathrm{kHz}$ 


\subsection{Impact of laser pulses per dot}

The experiments show a clear dependency of the achieved ablation depth and width on the applied pulses per dot. The number of the pulses per dot $N$ can be calculated using Equation 4 and is mainly influenced by the focal diameter $\mathrm{W}_{\mathrm{f}}$, the applied scan velocity $v_{\text {scan }}$ and pulse repetition rate $f_{\text {rep }}$.

$$
N=w_{f} \cdot \frac{f_{\text {rep }}}{v_{\text {scan }}}
$$

Another common way to describe the number of pulses per dot is using the pulse overlap PO. Equation 5 shows the correlation between $\mathrm{N}$ and $\mathrm{PO}$.

$$
P O=\left(1-\frac{1}{N}\right) \cdot 100 \%
$$

The threshold fluence $F_{t h}$ or ablation threshold of the material depends on the number of the applied pulses per dot and lowers with an increasing number of pulses. This effect is known as the incubation effect and can be calculated using Equation 6.

$$
F_{t h}(N)=F_{t h}(1) \cdot N^{S-1}
$$

In this model, $S$ represents a material- and crystal orientation-dependent parameter which is in the range of $0-1$. The more impact the number of pulses has on the ablation threshold, the closer $S$ is to 1 .

For the additive manufactured AlSi10Mg0.4 cubes the determined threshold fluences for a different number of pulses per dot are in a range of $0.34-0.6 \mathrm{~J} / \mathrm{cm}^{2}$, see Table 3 . It can be seen that for $N=9$ and $N=18$ there is no significant influence of the pulse repetition rate anymore. A difference for $\mathrm{N}<5$ might be caused by statistical measuring fault.

By double-logarithmic plot of $N \cdot$ Fth(N) over $N$, the incubation parameter $S$ can be determined from the slope of the linear fit. With this method and the experimental results a value for $S=0,78$ can be determined for laser wavelength of $1030 \mathrm{~nm}$ resulting in single pulse threshold fluence $F_{t h}(1)=0.6 \mathrm{~J} / \mathrm{cm}^{2}$

Table 3 Ablation threshold depending on the applied laser pulses per dot, exemplary for $\mathrm{t}_{\mathrm{H}}=0.8 \mathrm{ps}$

\begin{tabular}{lll|lll}
\hline $\mathrm{N}$ & $\begin{array}{l}\mathrm{PO} \\
{[\%]}\end{array}$ & $\begin{array}{l}\mathrm{F}_{\mathrm{th}(100 \mathrm{kHz})} \\
{\left[\mathrm{J} / \mathrm{cm}^{2}\right]}\end{array}$ & $\mathrm{N}$ & $\begin{array}{l}\mathrm{PO} \\
{[\%]}\end{array}$ & $\begin{array}{l}\mathrm{F}_{\mathrm{th}(200 \mathrm{kHz})} \\
{\left[\mathrm{J} / \mathrm{cm}^{2}\right]}\end{array}$ \\
\hline 2 & 50 & 0.51 & 4 & 75 & 0.56 \\
5 & 80 & 0.45 & 9 & 88 & 0.43 \\
9 & 88 & 0.44 & 18 & 94 & 0.37 \\
18 & 94 & 0.32 & 36 & 97 & 0,32 \\
\hline
\end{tabular}

Compared to the values of the ablation thresholds for pure aluminum $0.124 \mathrm{~J} / \mathrm{cm}^{2}$ [20], respectively $0,19 \mathrm{~J} / \mathrm{cm}^{2}$ [21]; or a comparable aluminum alloy AlMgSil with $0.3 \mathrm{~J} / \mathrm{cm}^{2}$ [22] the threshold fluence for AlSi $10 \mathrm{Mg} 0.4$ is more than doubled for a single pulse ablation. However, for a higher number of pules the threshold fluence is almost the same range as reported in literature.

As the ablation threshold decreases with a higher number of applied pulses per dot a higher ablation depth can be observed for lower scanning speeds. Figure 5 shows this behaviour exemplary for a fluence of $1.57 \mathrm{~J} / \mathrm{cm}^{2}$, which is representable for the regime of the dominant optical penetration depth, and a fluence of $3.93 \mathrm{~J} / \mathrm{cm}^{2}$, which represents the regime where the thermal diffusion length is significant.

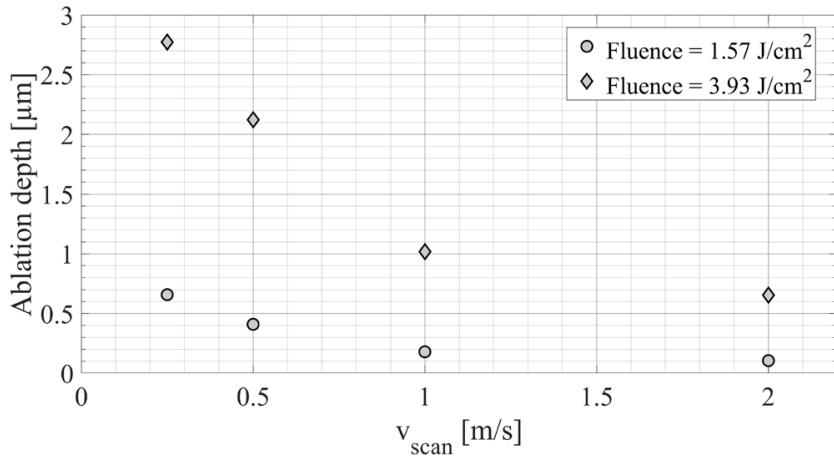

Fig. 5 Ablation depth depending on the applied fluence and scan velocity, exemplary for $\mathrm{f}_{\text {rep }}=100 \mathrm{kHz}$ and $\mathrm{t}_{\mathrm{H}}=0,8 \mathrm{ps}$

\subsection{Surface morphology}

Next to the ablation depth and width also the surface morphology of the ablated grooves plays an important role. For optical structures they should be as smooth as possible to prevent a scattering of the light by inhomogeneity. The achieved surface therefore is investigated on a macro scale using WLI images, see Figure 6 and on a micro scale using SEM images, see Figure 7.

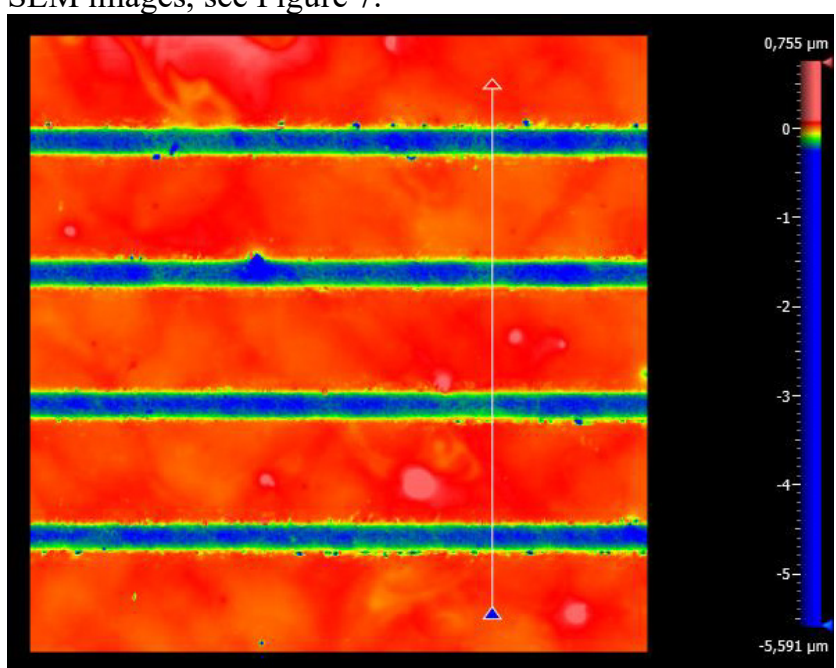

Fig. 6 WLI image of homogeneous ablated grooves with $\mathrm{V}_{\text {scan }}=0,5 \mathrm{~m} / \mathrm{s} ; \mathrm{t}_{\mathrm{H}}=0,8 \mathrm{ps}$ and $\mathrm{f}_{\mathrm{rep}}=200 \mathrm{kHz}$

It can be observed that for higher fluences a secondary structure, smaller than the focal spot itself occurs in the groove. This happens for fluences higher than $2.4 \mathrm{~J} / \mathrm{cm}^{2}$. Here, the ablation depth increases dramatically, whereas the ablation width starts to reduce again. Figure 7 shows the formation of the secondary structure due to melting of the material for three different pulse durations. The formation of melt also increases for a longer pulse duration which also is due to thermal effects and well known in literature. For aluminum the transition between a non-thermal ablation process and a thermal ablation process is reported to be between $0.7-5$ ps [23], always dependent on the applied fluence. As the selected parameter are covering most of this range, we are not able to achieve a complete non thermal ablation process for all fluences. However, we achieved a 
non-thermal ablation process if using low fluences. As example, the pictures a) - d) show clear and homogeneous ablated grooves, whose cross sections correspond to the Gaussian intensity distribution in the laser spot. In The pictures e) $-\mathrm{h}$ ) higher fluences were applied so even for the shortest used pulse duration of $0.8 \mathrm{ps}$ a formation of melting droplets can be observed after approx. $10 \mu \mathrm{m}$ distance from each edge. Further, this effect increases with longer pulse durations because of the longer laser-matter interaction times which offers more time for head conduction effects and melt generation.
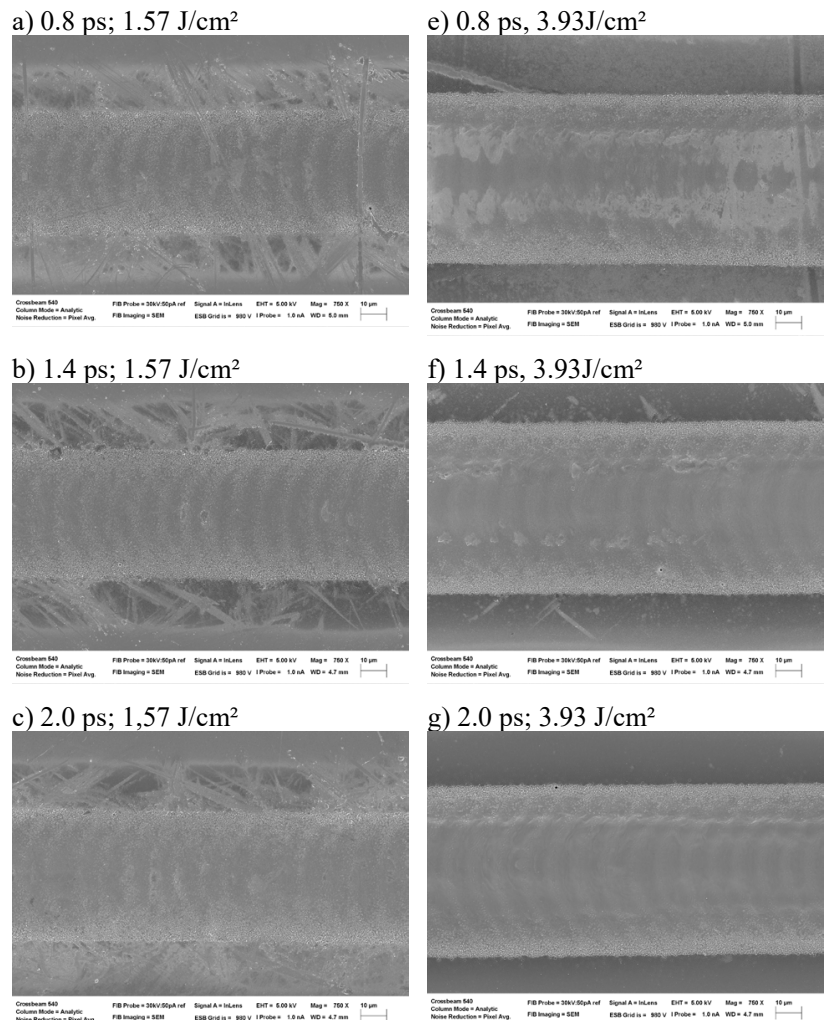

d) exemplary cross section of the WLI profile: $2.0 \mathrm{ps} ; 1.57 \mathrm{~J} / \mathrm{cm}^{2}$
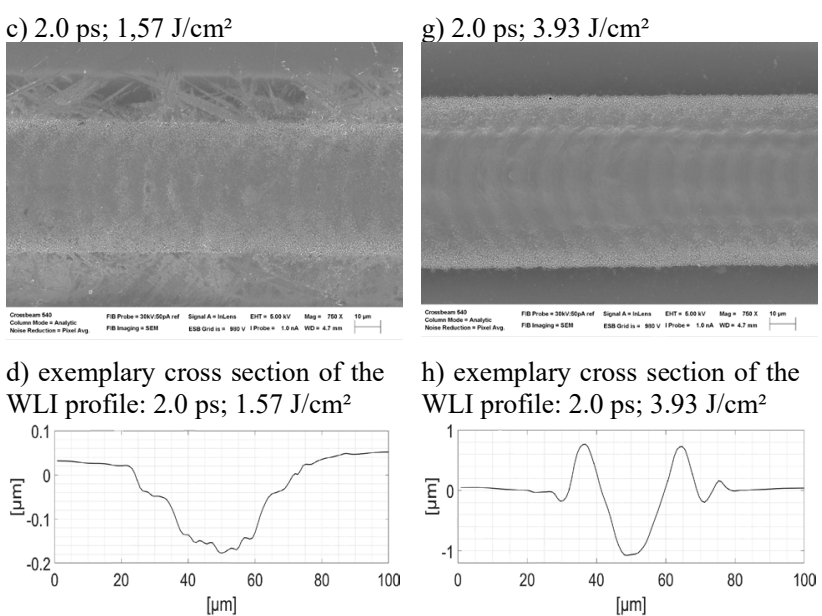

h) exemplary cross section of the WLI profile: $2.0 \mathrm{ps} ; 3.93 \mathrm{~J} / \mathrm{cm}^{2}$

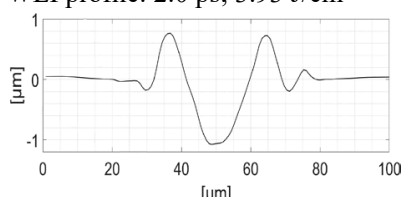

Fig. 7 Comparison between low fluences /clear ablation and high fluences / melt formation for different pulse durations at $\mathrm{f}_{\text {rep }}=100$ $\mathrm{kHz}$ and $\mathrm{v}_{\mathrm{s}}=1 \mathrm{~m} / \mathrm{s}(\mathrm{N}=5 ; \mathrm{PO}=79 \%)$. Top view SEM images and bottom view WLI cross sections.

In addition to the melt formations also an area with redeposited material can be observed, see e.g. Figure 7 a). The size of this area increases with the ablation volume. In contrast to Figure 7 a) this area out of the field of view for Figure 7 e) due to the high magnification. Figure 8 therefore shows a lower Magnification of field Figure 7 e). It can be clearly seen that this polluted area next to the ablated grove also exists and even enlargers if higher fluences are applied. Figure 8 also shows near surface pores. The pores are a result out of the additive manufacturing building process and the existing melt pool dynamics there.

The mechanical pretreatment before the ablation experiments closes them by filling the pores with removed material in the grinding process. However, they still exist and are just filled near the surface. During the ablation process these pores were opened again, which could lead to a very inhomogeneous ablation result along the scanned line.

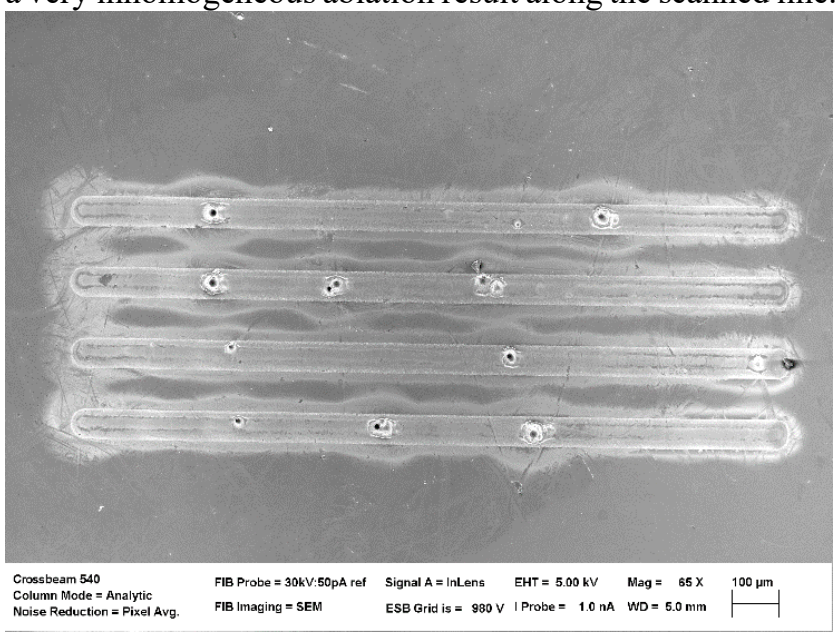

Fig. 8 Track overview with redeposition areas beside the grooves. $\mathrm{f}_{\text {rep }}=100 \mathrm{kHz} ; \mathrm{v}_{\mathrm{s}}=1 \mathrm{~m} / \mathrm{s}(\mathrm{N}=5 ; \mathrm{PO}=79 \%), \mathrm{F}=3.93 \mathrm{~J} / \mathrm{cm}^{2} ; 0.8$ ps

\section{Conclusion and Outlook}

Ultra-short pulsed laser direct structuring of additive manufactured AlSi10Mg0.4 parts offers a precise postprocessing method. However, to achieve a highly homogenous surface structure as required for optical applications, a direct structuring process on the surface as produced is not possible. Just using common laser ablation as only post-processing method provides a not satisfying result to determine ablation thresholds and clear ablation grooves. In combination with conventional mechanical grinding the results of the experiments show a clear dependency of the input fluence and the achieved ablation depth. Further, the ablation depth strongly depends on the scan velocity and pulse repetition rate, respectively the resulting pulse overlap, as well as on the used pulse duration which is a clearly hint for incubation effects on AlSil0Mg0.4. With the help of the experiments, the incubation parameter $S$ could be determined and a pulse duration dependency for $S$ could not be shown.

Although normally femtosecond laser pulses are taken for ablation the experiments have shown that the used laser source has enough power to create a melting of the material. This is why the future work will focus on ablation experiments using a combined laser treatment, e.g. using defocused high energy pulses to polish the surface with the same laser beam before ablation. This procedure might be a promising solution to allow a quasi-direct laser structuring on the additive manufactured surface as produced. Further, if near surface pores can be closed by the laser polishing process before the ablation, more homogenous ablation result will be reached. A pore free ablation grove will be necessary to e.g. use direct laser ablation as a process for the fabrication of reflection gratings on additive manufactured mirrors. 


\section{Acknowledgments}

The authors would like to thank Prof. Dr. Andreas Heinrich from the Center of Optical Technologies at Aalen University, David Kolb and Tim Schubert for their support. We acknowledge support by the German Federal Ministry of Education and Research, program 'FH-Impuls' (AddFunK, grant no. 03FH4I04IA) and 'FH-Invest (FlexLight 4.0 grant no. $13 \mathrm{FH} 114 \mathrm{~N} 6)$.

\section{References}

[1] D.R. Eyers and A.T. Potter: Comput. Ind., 92-93, (2017) 208.

[2] M. Brandt: "Laser additive manufacturing", Materials design technologies and applications (Elsevier Inc, Duxford, 2017).

[3] M. Schneck, M. Gollnau, M. Lutter-Günther, B. Haller, G. Schlick, M. Lakomiec, G. Reinhart: Proc. CIRP, 81, (2019) 19.

[4] A. Heinrich and M. Rank: "3D Printing of Optics", (SPIE Press, Bellingham, 2018).

[5] F. Niesler and Y. Tanguy: Optik \& Photonik, 11, (2016) 44

[6] T. Gissibl, S. Thiele, A. Herkommer, H. Giessen: Nat. Commun., 7, (2016) 11763.

[7] A. Heinrich, R. Börret, M. Merkel, H. Riegel: Proc. SPIE, Vol. 10523, (2018) 1052302.

[8] E. Hilpert, J. Hartung, H. von Lukowicz, T. Herffurth, N. Heidler: Opt. Eng., 58, (2019) 092613.

[9] N. Heidler, E. Hilpert; J. Hartung, T. Albert: Conf. Proc. EUSPEN, (2017) "Lightweight structures for metal mirrors".

[10] H.E. Bennett, J.M. Bennett and E.J. Ashley: J. Opt. Soc. Am., 52, (1962) 11.

[11] G. Hass and J.E. Waylonis: J. Opt. Soc. Am., 51, (1961) 7 .

[12] N. Heidler, E. Hilpert, J. Hartung, H. von Lukowicz, C. Damm, T. Peschel, S. Risse: Proc. SPIE, Vol. 10692, (2019), 106920C.

[13] T. Sugano, K. Takeuchi, T. Goto, Y. Yoshida, N. Ikawa: CIRP Annals, 36, (1987) 1.

[14] R. Almeida, R. Börret, M. Pohl, D.K. Harrison, A. De Silva: Prod. Manuf. Res., 6, (2018) 247.

[15] J. Schanz, M. Hofele, S. Ruck, T. Schubert, L. Hitzler, G. Schneider, M. Merkel, H. Riegel: Materwiss Werksttech., 48, (2017) 463.

[16] J. Schmidt, R. Scholz and H. Riegel.: Materwiss Werksttech., 46, (2015) 686.

[17] I. Mingareev, N. Gehlich, T. Bonhoff, W. Meiners, I. Kelbassa, T. Biermann, M.C. Richardson: Proc. SPIE, Vol. 8970, (2014) 89700R.

[18] N. Worts, J. Jones and J. Squier: Opt. Commun., 430, (2019) 352.

[19] I. Mingareev, T. Bonhoff, A.F. El-Sherif, W. Meiners, I. Kelbassa, T. Biermann, M.C. Richardson: J Laser Appl., 25, (2013) 5.

[20] S. Weiler, U. Stute, S. Massa, S. Buettner, B. Faisst: Proc. SPIE, Vol. 6881, (2008) 68811B.

[21] R. Le Harzic, D. Breitling, M. Weikert, S. Sommer, C. Fhl, F. Dausinger, S. Valette, C. Donnet, E. Audouard: Appl. Phys. A, 80, (2005) 7.

[22] J. Krüger, P. Meja, M. Autric, W. Kautek: Appl. Surf. Sci., 186, (2002) 1.
[23] M. Weikert:“Oberflächenstrukturieren mit ultrakurzen Laserpulsen” (Utz, München, 2006).

(Received: June 23, 2020, Accepted: February 1, 2021) 